Unfallchirurg 2009 $\cdot 112: 233$

DOI 10.1007/s00113-009-1611-4

(c) Springer Medizin Verlag 2009

\author{
C. Krettek \\ Unfallchirurgische Klinik, Medizinische Hochschule Hannover
}

\title{
Leitlinien zur kardiopulmonalen Reanimation
}

\section{Was ist wichtig für den Unfallchirurgen?}

Die International Liaison Committee (ILCOR) hat im Jahr 2005 die derzeit gültigen Leitlinien zur kardiopulmonalen Reanimation veröffentlicht. Diese Leitlinien beinhalten $u$. a. aktualisierte Empfehlungen zum sensiblen Bereich der lebensrettenden Maßnahmen bei Kindern. Bei der Erstellung der Leitlinien für Kinder wurde ein besonderes Augenmerk auf eine möglichst weitgehende Vereinfachung der Behandlungsabläufe gelegt, da insbesondere durch Ersthelfer aufgrund von Unsicherheiten und Ängsten häufig erst spät Reanimationsmaßnahmen eingeleitet werden. Eine offensichtliche Änderung ist die Aufhebung der bisherigen Unterscheidung in Kinder über und unter 8 Jahren. Die Anwendung der pädiatrischen Leitlinien wird nun vielmehr bis zu dem Beginn der Pubertät empfohlen, da dieser Zeitpunkt eine der augenfälligsten Veränderungen in der Entwicklung eines Menschen darstellt. Es wird ebenso weiterhin zwischen Säuglingen ( $<1$ Jahr) und älteren Kindern unterschieden, da es auch hier wichtige Unterschiede zwischen diesen Altersgruppen gibt. Allerdings erscheint auch in den neuen Leitlinien aufgrund wesentlicher Unterschiede zwischen dem bei Erwachsenen vorherrschenden kardial bedingten Stillstand und dem bei Kindern sehr häufig vorkommenden asphyktischen Stillstand bei professionellen Helfern weiterhin ein spezieller Kinderalgorithmus gerechtfertigt.

Neben einem asphyktischen und kardial bedingten Stillstand können weitere Ursachen zu einer Reanimationspflichtigkeit eines Patienten führen. Diese Umstände, die für das behandelnde Medizinpersonal zur erfolgreichen Behandlung des Patienten essenziell sind, werden in den Leitlinien des ILCOR im Kapitel „Kreislaufstillstand unter besonderen Umständen “ analysiert. So können Elektrolytstörungen, hier insbesondere eine Dysregulation des Kaliumhaushaltes, zu einem Herz-Kreislauf-Stillstand führen. Der Kreislaufstillstand aufgrund von Vergiftungen durch Medikamente oder Drogen ist insgesamt selten, stellt aber eine Hauptursache bei Betroffenen unter 40 Jahren dar. Weitere Aspekte dieses Kapitels der ILCOR-Leitlinien sind Reanimationsmaßnahmen aufgrund von Ertrinkungsunfällen, Anaphylaxie, Hypothermie oder schweren Asthmaanfällen.

Für den Unfallchirurgen besonders interessant dürfte der traumatisch bedingte Kreislaufstillstand sein, der mit einer Gesamtüberlebensrate von ca. $2 \%$ eine sehr hohe Mortalität aufweist. Unter $1 \%$ der überlebenden Patienten ist neurologisch unauffällig.

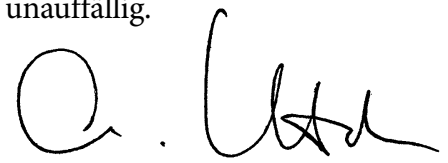

C. Krettek

\section{Korrespondenzadresse \\ Prof. Dr. C. Krettek \\ Unfallchirurgische Klinik, \\ Medizinische Hochschule Hannover \\ Carl-Neuberg-Straße 1, 30625 Hannover \\ krettek.christian@mh-hannover.de}

Interessenkonflikt. Keine Angaben. 